\title{
The Research Progress of Long Noncoding RNAs in Autoimmune Diseases
}

\author{
Li Zou, Md Rezaul Karim and Yun-fu Wang*
}

Department of Neurology, Taihe Hospital, Hubei University of Medicine, Shiyan, Hubei 442000, P. R. China

*Corresponding author: Yun-fu Wang, Department of Neurology, Taihe Hospital, Hubei University of Medicine, Shiyan, Hubei 442000, P. R. China, E-mail: wyfymc@163.com

Received date: January 18, 2016; Accepted date: March 09, 2016; Published date: March 16, 2016

Copyright: (C) 2016 Karim RM, et al. This is an open-access article distributed under the terms of the Creative Commons Attribution License, which permits unrestricted use, distribution, and reproduction in any medium, provided the original author and source are credited.

\begin{abstract}
Long Noncoding RNAs (IncRNAs) are transcribed RNAs molecules greater than 200 nucleotides in length. Nowadays, IncRNAs are found to have lots of functions including regulating transcription, post-transcriptional RNA processing, translation, gene rearrangement and chromatin modification. Abnormal expressions and functional abnormity of IncRNAs may play an important role in the development and progress of autoimmune diseases. Thus a systematic and comprehensive profiling of IncRNA will help us to have a deeper understanding of the pathogenesis of the diseases. This article will focus on the latest research progress of IncRNA in autoimmune diseases, which will contribute to clinical applications for diagnosis, prognosis and treatment of autoimmune diseases.
\end{abstract}

Keywords: Long Noncoding RNAs; Autoimmune diseases; Research strategy; Systemic lupus erythematosus; Rheumatoid arthritis; Multiple sclerosis; Psoriasis; Sjogren’s syndrome

\section{Introduction}

Over the past few decades research has generally focused on the protein encoding gene. However in recent years, through genome-wide transcriptional analysis research shows that transcription without choice of mammalian genomes may be common. There are a variety of codes of noncoding RNA (ncRNA) [1,2]. Prior to a period of time within noncoding transcription has been considered junk DNA and transcription of "noises" [3]. However recent studies indicate that, these ncRNA are also involved in the maintenance of tissue homeostasis and other physiological processes of nucleus [4-6]. According to its size ncRNAs can be roughly divided into two main categories: small ncRNAs ( $<200 \mathrm{nt})$, such as micro RNA (miRNAs); long chain ncRNA ( $\geq 200 \mathrm{nt}$ ). In the pathogenesis of inflammatory and autoimmune diseases, miRNA has been shown to be an important regulatory factor of numerous genes and signaling pathways, such as Systemic lupus erythematosus (SLE), Rheumatoid arthritis (RA), Psoriasis (Systemic sclerosis, SSc), Primary Sjogren's syndrome (SS) and Multiple sclerosis (MS) [7,8]. Recently ncRNAs in another major category, long noncoding RNA (lncRNAs) has been widespread concern in the field of molecular biology. A growing body of evidence suggests that this type of RNA can be processed protein coding mRNA and participate in a variety of physiological and biological processes such as cell proliferation, differentiation, apoptosis and immune response [9-13]. Previous studies have found that, lncRNAs focused on exploring the relationship between genomic imprinting, cancer and cell differentiation. On the other hand, current studies have found lncRNAs in innate and acquired immune system, differentiation and activation of immune cells, which play an important regulatory role. This suggests that, lncRNA play a key role in the autoimmune process and autoimmune diseases. In this review, we will briefly introduce the biological effects of lncRNA and summarizes its development in pathogenesis, representing several autoimmune diseases in the current research progress.

\section{Overview of long noncoding RNA}

Long noncoding RNA (lncRNA) is longer than the RNA molecule $200 \mathrm{nt}$; itself does not encode proteins with a specific secondary structure, expression of tissue specificity and temporal specificity, often located in the nucleus or inside cytoplasm in the form of RNA in gene transcription regulation, epigenetic level, chromatin modification, post-transcriptional regulation and other multi-level, multiparticipatory biological processes. Recent studies have found, lncRNA general transcription, mutation and regulatory function abnormalities can cause a variety of human diseases [14]. Brief description of the classification and functions LncRNAs are following.

According to lncRNA relative position in the genome encodes protein is mainly divided into five categories: (1) justice lncRNA (2) an antisense lncRNA, both separately are protein-coding sequence of one or more exons encoding forward, reverse overlap (3) two-way lncRNA, refers to this lncRNA expression and protein-coding sequence adjacent to a promoter sequence and is located on the antisense strand, both opposite direction of transcription (4) gene lncRNA, it refers to its sequence entirely by another transcript intron derived lncRNA (5) between gene lncRNA, is located between two genes means independent, not associated with any protein-coding gene sequences transcribed from neighboring lncRNA $[15,16]$. Most characteristic lncRNA are not exactly accurate classification within the genome. According to their characteristics, these lncRNA are: activated lncRNA (lncRNA-a) gene, ultra-conserved elements gene (pseudogene), telomere associated noncoding RNA (TERRAs), transcription (TUCRs), enhancer RNAs (eRNAs), ring-type RNA [17-20]. These noncoding transcripts often associated with the coding region of the corresponding mRNA or minimal overlap, whether or not it may encode proteins, but these mRNA has its inherent function. LncRNAs has been involved in a variety of ways by a wide range of biological functions, but there is still little known about the molecular mechanism. We understand, LncRNAs major genetic influences can output within genes almost every stage of the life cycle, from remodeling and epigenetic regulation of chromatin, transcriptional and post-transcriptional regulation to protein metabolism [21,22]. Part lncRNAs involved in chromatin remodeling complex formation and 
Page 2 of 4

mediated gene silencing [23]. However lncRNAs recycling is achieved within more binding regulatory proteins, RNA and DNA interactions probably by modularity [24]. It also can be used as noncoding DNA which is inherited evolutionary conservation of the transcript, this effect may be related to regulation of cell differentiation [25], genomic rearrangements and inactivation of tumor suppressor genes [4]. LncRNAs has been confirmed, it can adjust the adjacent (cis-acting) within a large number of genomes or remote (trans-acting) of the gene product, or trans-acting transcription-dependent transcription lncRNA called cis-acting lncRNA dependence [26]. Recently, Marques and Ponting nucleus by the appropriate organizations LncRNA genomic features were set target screening and found that most low Shunyi LncRNA characteristic variation while antisense LncRNA characteristic variability may be higher [27]. In addition, a variety of molecular mechanisms and biological functions LncRNA is relevant [28], but research is still limiting its precise understanding of specific mechanisms. Therefore, LncRNAs dysfunctional performance and a variety of human polygenic diseases from cancers of different organs [29] to non-cancerous diseases, such as Alzheimer's disease [30], coronary heart disease [31], myocardial infarction [32] and $\mathrm{X}$ chromosome inactivation transcribed (Xist) membranous nephropathy [33]. Meanwhile, LncRNA congenital or acquired immune system are affected alternately, their further research may help us better understand infectious and inflammatory diseases $[34,35]$.

\section{Long noncoding RNA and autoimmune diseases}

Over time, autoimmune disease progressed by interaction of genetics and environmental factors, arising from a complex disease. Although significantly progressed the new treatments but for many patients, the long-term effect is still not ideal [36-38]. Therefore, we need a better understanding of the etiology of autoimmune diseases. There are many types of autoimmune diseases, such as: Systemic lupus erythematosus (SLE), Rheumatoid arthritis (RA), Multiple sclerosis (MS), Psoriasis and Sjogren's syndrome (SS) etc. Although autoimmune diseases have significant clinical differences, but there are many clinical features and pathogenesis those are substantially the same. For example: SLE, RA, SS and Psoriasis have a chronic inflammatory joint symptoms [39], SLE and SSc exist within the cycle of anti-intracellular antigens autoantibodies; such as antinuclear antibodies (ANAs) [40]. Currently, LncRNAs regulate normal immune function in a variety of evidence that is quite more. However it is not yet in-depth understanding that, lncRNAs disorder is associated with the pathogenesis of autoimmune diseases. But new evidence has shown that, LncRNAs dysfunction may in autoimmune diseases such as SLE, RA, MS, SS etc., which play a key role $[41,42]$.

\section{Long noncoding RNA and Systemic Lupus Erythematosus}

Systemic lupus erythematosus (SLE) is a typical which is involving in congenital and acquired sophisticated interactivity between systemic autoimmune disease of the immune system, which is lost since nuclear antigen immune tolerance and produce autoantibodies [43]. The main feature of this disease is to produce anti double-stranded DNA (dsDNA) autoantibodies and anti-nuclear autoantigens, other autoantibodies. Despite a lot of research, but there are still many mysteries cause of SLE is now recognized: genetic and epigenetic predisposition; as well as familiar and unfamiliar environmental factors play a pivotal role in the development of SLE [44]. Recently, Genome-wide association studies (GWAS), has identified chromosome 1q25 region which are associated with SLE. A Long noncoding RNA called GAS5 may be a prime candidate for chromosome 1q25 locus associated with SLE. Thus, genetic evidence suggests that: GAS5 is remainder of SLE susceptibility $[23,45]$. In addition, in a mouse model, GAS5 increased SLE susceptibility, suggesting that the results may influence the effect of glucocorticoids as immunosuppressive agents [23]. The loss of GAS5 promoter Sp1 binding sites can result in low expression of GAS5. So, author guess GAS5 may inhibit cell cycle and apoptosis, which may be exposed and autoantibody production by promoting antigen, involving the incidence of autoimmune diseases [46]. In a recent study, Shi and others in their small sample study, nine cases of female SLE patients monocytes were purified whole transcriptome analysis, gene expression and by comparison with eight healthy control group; they have found that the presence of specific selectivity SLE splicing, alternative polyadenylation, transcription of new sites and reproducible effect on the control monocytes LPS treatment [47]. In addition, the study confirmed that, the carrier in a mouse model of type I interferons, the non-coding RNA expression Aicardi Goutieres syndrome is reduced. Primary miRNA (Pri-miRNA) is clearly induced in the pathogenesis of SLE; these small non-coding RNA is processed to inhibit transcription and regulation of several specific Messenger RNA. Compared with the healthy control group; there are two specific pri-miRNA in SLE monocytes which is significantly increased and the two of miRNA message level is declining, suggesting that pri-miRNA in SLE monocytes are functionally -related. LncRNAs compared additionally to other types of RNA - is unlikely to change in SLE, while those with a significant change in the position of suggesting that, LncRNA involved in the pathogenesis of SLE development. For example, Human immunodeficiency virus type I enhancer binding protein-2 (HIVEP2) itself and an LncRNA (about 800-1500 base) are in the Transcription start site (TSS) upstream of expression in SLE were significantly upregulated. In SLE monocytes, located in LncRNAs on chromosome 6q25.3 has generally offset [41].

\section{Long noncoding RNA and Psoriasis}

Psoriasis is a chronic skin and joint manifestations of hyperproliferative diseases, currently affecting the normal life about $2-3 \%$ of the total population [48]. Thickening of the skin of patients in psoriasis is mainly due to abnormal basal keratinocyte proliferation and differentiation of cells [49]. Changes in gene expression of Psoriasis have been well documented. In addition to protein-coding gene has its own characteristic features, Long non-coding RNA a newly discovered - PRNS (under stress-induced psoriasis-related non-protein-coding RNA), has been demonstrated in patients with psoriasis is not exists in the epidermis, and psoriasis in contrast skin lesions and normal skin are highly expressed; this indicates that PRINS may play an important role in psoriasis susceptibility rather than in psoriatic lesions from the skin to maintain the role [50]. Moreover, some studies have shown that, PRINS play a protective role [50-52] in cellular stress reactions. In addition, Szegedi [53], who found that, PRINS over expressed in psoriasis and adjust G1P3. G1P3 is an anti-apoptotic gene in cancer cells. High expression of G1P3 may help to maintain the development of psoriatic lesions keratinocyte hyperplasia cells, which assist the progress of psoriatic lesions [53]. Therefore, these studies show that, in the epidermis, elevated expression levels of PRINS may play an important role in psoriasis. In addition, RNA interference can reduces RNA PRINS after serum starvation level, but not in normal serum conditions on cell viability. It is proven that, PRINS can also act as "riboregulator" to manage the expression of other genes involved in cell display stress proliferation and survival. Another lncRNA gene, was 
named PSORS1C3 (psoriasis susceptibility 1 candidate 3); its closer HLA-C, and is located within the PSORS1 sites may also be psoriasis susceptibility gene [54]. To 178 Chinese people with psoriasis vulgaris patients PSORS1C3 correlation analysis of whole genome, we can confirm PSORS1C3 patients with psoriasis vulgaris gene is an important psoriasis susceptibility genes [55].

\section{Long noncoding RNA and Sjogren's syndrome}

Sjogren's syndrome (SS) is an autoimmune disease characterized by major exocrine glands, such as salivary, lacrimal gland invasion by inflammation, leading to dry mouth, dry eyes and other symptoms [56]. Age of onset is usually 40-50 years old and mostly is women. SS logo is the performance of B-cell function hyperthyroidism, such as hypergammaglobulinemia, circulating immune complexes and antiRo / SSA, anti-La / SSB autoantibodies [57]. In the minor salivary glands (MSG) RNA in a sample study, primary SS may be made Coxsackie B4 virus (CVB4) p2A gene 94bp fragment length; this study may clarify that, CVB4 may induce and maintain effects of primary Sjogren's syndrome [58]. However, in PubMed and MeSH database, Sjogren's syndrome research lncRNA cannot be retrieved; research shows there is still a great prospect.

\section{Long noncoding RNA and Multiple Sclerosis}

Multiple sclerosis (MS) is characterized by chronic inflammatory demyelinating, oligodendrocytes apoptosis and axonal degeneration. Burfoot [59], who demonstrated that, HLA-A*2 and $A^{\star} 3$ multiple sclerosis plays an important role in sensitivity, and myelin oligodendrocyte glycoprotein (MOG) regulate gene HLA-DRB1 complete after two encoded multi-normality which also have an important influence on multiple sclerosis susceptibility. Often due to infection TMEV, an important means of experimental mouse model of multiple sclerosis. Therefore, the role of T cell regulation IFNG-AS1 (Tmevpg1) is considered to be involved in another important means in lncRNA multiple sclerosis disease development [60]. However, lncRNA research in multiple sclerosis more or less, the only research is still at the primary level.

\section{Conclusion}

All in all, LncRNA is longer than 200 nt RNA and RNA does not encode proteins, plays an important role in autoimmune diseases such as: Systemic lupus erythematosus, Rheumatoid arthritis, Psoriasis, etc., and a variety of multiple genes in human diseases. Immune cell activation, differentiation and unbalanced expression, T cells, B cells and NK cells in autoimmunity can change is directly linked with lncRNA [41]. However in autoimmune diseases, identification of functional performance of lncRNA still needs to study furthermore. For example, why lncRNA expression in autoimmune diseases is exceptions and what is the specific mechanism? How in the etiology and pathogenesis of autoimmune diseases distinguish lncRNAs and other non-coding RNA, or other association epigenetic factor? In addition, it is unclear whether the change lncRNAs plays a causal role in disease progression, whether manipulation of lncRNAs can play a beneficial effect in the treatment of autoimmune diseases? Most of the transcribed noncoding DNA can encode RNA. The relative proportions of noncoding genomic DNA increases the complexity of the development, which indicates that more ncRNA may have their important biological functions as well as in autoimmune diseases like cancer in different organs. In near future, further research will be both in vitro and in vivo application of advanced molecular biology techniques, such as: gene chips and next generation gene sequencing. This will reveal the mysteries disease regulation of lncRNA. Overall, studies of functional lncRNA biology and autoimmune diseases can further deepen the development of autoimmune diseases and understanding of the pathogenesis [41]. In near future, further studies of lncRNAs may contribute to the clinical diagnosis of autoimmune diseases [42]; as well as its clinical application may open up a whole new range of opportunities for treatment [61].

\section{References}

1. Djebali S, Davis CA, Merkel A, Dobin A, Lassmann T, et al. (2012) Landscape of transcription in human cells. Nature 489: 101-108.

2. ENCODE Project Consortium (2012) An integrated encyclopedia of DNA elements in the human genome. Nature 489: 57-74.

3. Wright MW, Bruford EA (2011) Naming 'junk': human non-protein coding RNA (ncRNA) gene nomenclature. Hum Genomics 5: 90-98.

4. Moran VA, Perera RJ, Khalil AM (2012) Emerging functional and mechanistic paradigms of mammalian long non-coding RNAs. Nucleic Acids Res 40: 6391-6400.

5. Ørom UA, Derrien T, Beringer M, Gumireddy K, Gardini A, et al. (2010) Long noncoding RNAs with enhancer-like function in human cells. Cell 143: 46-58.

6. Sun L, Goff LA, Trapnell C, Alexander R, Lo KA, et al. (2013) Long noncoding RNAs regulate adipogenesis. Proc Natl Acad Sci U S A 110: 3387-3392.

7. Singh RP, Massachi I, Manickavel S, Singh S, Rao NP, et al. (2013) The role of miRNA in inflammation and autoimmunity. Autoimmun Rev 12: 1160-1165.

8. Jimenez S A, Piera-Velazquez S (2013) Potential role of human-specific genes, human-specific microRNAs and human-specific non-coding regulatory RNAs in the pathogenesis of systemic sclerosis and Sjogren's syndrome. Autoimmun Rev 12: 1046-1051.

9. Kretz M, Webster DE, Flockhart RJ, Lee CS, Zehnder A, et al. (2012) Suppression of progenitor differentiation requires the long noncoding RNA ANCR. Genes Dev 26: 338-343.

10. Feldstein O, Nizri T, Doniger T, Jacob J, Rechavi G, et al. (2013) The long non-coding RNA ERIC is regulated by E2F and modulates the cellular response to DNA damage. Mol Cancer 12: 131.

11. Kretz M, Siprashvili Z, Chu C, Webster DE, Zehnder A, et al. (2013) Control of somatic tissue differentiation by the long non-coding RNA TINCR. Nature 493: 231-235.

12. Ying L, Huang Y, Chen H, Wang Y, Xia L, et al. (2013) Downregulated MEG3 activates autophagy and increases cell proliferation in bladder cancer. Mol Biosyst 9: 407-411.

13. Wang P, Xue Y, Han Y, Lin L, Wu C, et al. (2014) The STAT3-binding long noncoding RNA lnc-DC controls human dendritic cell differentiation. Science 344: 310-313.

14. Wapinski O, Chang H Y (2011) Long noncoding RNAs and human disease. Trends Cell Biol 21: 354-361.

15. Mercer TR, Dinger ME, Mattick JS (2009) Long non-coding RNAs: insights into functions. Nat Rev Genet 10: 155-159.

16. Ponting CP, Oliver PL, Reik W (2009) Evolution and functions of long noncoding RNAs. Cell 136: 629-641.

17. Ledford H (2013) Circular RNAs throw genetics for a loop. Nature 494: 415.

18. Mercer TR, Wilhelm D, Dinger ME, Soldà G, Korbie DJ, et al. (2011) Expression of distinct RNAs from 3' untranslated regions. Nucleic Acids Res 39: 2393-2403.

19. Smemo S, Campos LC, Moskowitz IP, Krieger JE, Pereira AC, et al. (2012) Regulatory variation in a TBX5 enhancer leads to isolated congenital heart disease. Hum Mol Genet 21: 3255-3263.

20. Fang XY, Pan HF, Leng RX, Ye DQ (2015) Long noncoding RNAs: novel insights into gastric cancer. Cancer Lett 356: 357-366. 
21. Schonrock N, Harvey RP, Mattick JS (2012) Long noncoding RNAs in cardiac development and pathophysiology. Circ Res 111: 1349-1362.

22. Shi X, Sun M, Liu H, Yao Y, Song Y (2013) Long non-coding RNAs: a new frontier in the study of human diseases. Cancer Lett 339: 159-166.

23. Kino T, Hurt DE, Ichijo T, Nader N, Chrousos GP (2010) Noncoding RNA gas5 is a growth arrest- and starvation-associated repressor of the glucocorticoid receptor. Sci Signal 3: ra8.

24. Guttman M, Rinn JL (2012) Modular regulatory principles of large noncoding RNAs. Nature 482: 339-346.

25. Cabili MN, Trapnell C, Goff L, Koziol M, Tazon-Vega B, et al. (2011) Integrative annotation of human large intergenic noncoding RNAs reveals global properties and specific subclasses. Genes Dev 25: 1915-1927.

26. Ulitsky I, Bartel DP (2013) lincRNAs: genomics, evolution, and mechanisms. Cell 154: 26-46.

27. Marques AC, Ponting CP (2014) Intergenic lncRNAs and the evolution of gene expression. Curr Opin Genet Dev 27: 48-53.

28. Kung JT, Colognori D, Lee JT (2013) Long noncoding RNAs: past, present, and future. Genetics 193: 651-669.

29. Li J, Xuan Z, Liu C (2013) Long non-coding RNAs and complex human diseases. Int J Mol Sci 14: 18790-18808.

30. Faghihi MA, Modarresi F, Khalil AM, Wood DE, Sahagan BG, et al. (2008) Expression of a noncoding RNA is elevated in Alzheimer's disease and drives rapid feed-forward regulation of beta-secretase. Nat Med 14: 723-730.

31. McPherson R, Pertsemlidis A, Kavaslar N, Stewart A, Roberts R, et al. (2007) A common allele on chromosome 9 associated with coronary heart disease. Science 316: 1488-1491.

32. Ishii N, Ozaki K, Sato H, Mizuno H, Saito S, et al. (2006) Identification of a novel non-coding RNA, MIAT, that confers risk of myocardial infarction. J Hum Genet 51: 1087-1099.

33. Huang YS, Hsieh HY, Shih HM, Sytwu HK, Wu CC (2014) Urinary Xist is a potential biomarker for membranous nephropathy. Biochem Biophys Res Commun 452: 415-421.

34. Fitzgerald KA, Caffrey DR (2014) Long noncoding RNAs in innate and adaptive immunity. Curr Opin Immunol 26: 140-146

35. Carpenter S, Aiello D, Atianand MK, Ricci EP, Gandhi P, et al. (2013) A long noncoding RNA mediates both activation and repression of immune response genes. Science 341: 789-792.

36. Cope AP, Feldmann M (2004) Emerging approaches for the therapy of autoimmune and chronic inflammatory disease. Curr Opin Immunol 16: 780-786.

37. Pan HF, Li XP, Zheng SG (2013) Emerging role of interleukin-22 in autoimmune diseases. Cytokine Growth Factor Rev 24: 51-57.

38. Chatenoud L (2006) Immune therapies of autoimmune diseases: are we approaching a real cure? Curr Opin Immunol 18: 710-717.

39. Kunz M, Ibrahim SM (2009) Cytokines and cytokine profiles in human autoimmune diseases and animal models of autoimmunity. Mediators Inflamm 2009: 979258.

40. Mahler M, Fritzler MJ, Satoh M (2015) Autoantibodies to the mitochondrial RNA processing (MRP) complex also known as Th/To autoantigen. Autoimmun Rev 14: 254-257.

41. Sigdel KR, Cheng A, Wang Y, Duan L, Zhang Y (2015) The Emerging Functions of Long Noncoding RNA in Immune Cells: Autoimmune Diseases. J Immunol Res 2015: 848790.

42. Wua BGC, Pana BHF, Lenga BRX, Wangc DG, Lid XP, et al (2015) 'Emerging role of long noncoding rnas in autoimmune diseases'. Autoimmun Rev 14: 798-805.
43. Liu CC, Kao AH, Manzi S, Ahearn JM (2013) Biomarkers in systemic lupus erythematosus: challenges and prospects for the future. Ther Adv Musculoskelet Dis 5: 210-233.

44. Rhodes B, Vyse TJ (2008) The genetics of SLE: an update in the light of genome-wide association studies. Rheumatology (Oxford) 47: 1603-1611.

45. Suarez-Gestal M, Calaza M, Endreffy E, Pullmann R, Ordi-Ros J, et al. (2009) Replication of recently identified systemic lupus erythematosus genetic associations: a case-control study. Arthritis Res Ther 11: R69.

46. Haywood ME, Rose SJ, Horswell S, Lees MJ, Fu G, et al. (2006) Overlapping BXSB congenic intervals, in combination with microarray gene expression, reveal novel lupus candidate genes. Genes Immun 7: 250-263.

47. Shi L, Zhang Z, Yu AM (2014) The SLE transcriptome exhibits evidence of chronic endotoxin exposure and has widespread dysregulation of noncoding and coding RNAs. PLoS One 9: e938-946.

48. Di Meglio P, Villanova F, Nestle FO (2014) Psoriasis. Cold Spring Harb Perspect Med 4.

49. Roberson ED, Bowcock AM (2010) Psoriasis genetics: breaking the barrier. Trends Genet 26: 415-423.

50. Sonkoly E, Bata-Csorgo Z, Pivarcsi A, Polyanka H, Kenderessy-Szabo A, et al. (2005) Identification and characterization of a novel, psoriasis susceptibility-related noncoding RNA gene, PRINS. J Biol Chem 280: 24159-24167.

51. Bari L, Bacsa S, Sonkoly E, Bata-Csörgo Z, Kemény L, et al. (2011) Comparison of stress-induced PRINS gene expression in normal human keratinocytes and HaCaT cells. Arch Dermatol Res 303: 745-752.

52. Szegedi K, Göblös A, Bacsa S, Antal M, Németh IB, et al. (2012) Expression and functional studies on the noncoding RNA, PRINS. Int J Mol Sci 14: 205-225.

53. Szegedi K, Sonkoly E, Nagy N, Németh IB, Bata-Csörgo Z, et al. (2010) The anti-apoptotic protein G1P3 is overexpressed in psoriasis and regulated by the non-coding RNA, PRINS. Exp Dermatol 19: 269-278.

54. Holm SJ, Sánchez F, Carlén LM, Mallbris L, Ståhle M, et al. (2005) HLA$\mathrm{Cw}^{\star} 0602$ associates more strongly to psoriasis in the Swedish population than variants of the novel 6p21.3 gene PSORS1C3. Acta Derm Venereol 85: 2-8.

55. Chang YT, Chou CT, Shiao YM, Lin MW, Yu CW, et al. (2006) Psoriasis vulgaris in Chinese individuals is associated with PSORS1C3 and CDSN genes. Br J Dermatol 155: 663-669.

56. Fox RI (2005) Sjögren's syndrome. Lancet 366: 321-331.

57. Gaidamakov S, Maximova OA, Chon $\mathrm{H}$, Blewett $\mathrm{NH}$, Wang $\mathrm{H}$, et al. (2014) Targeted deletion of the gene encoding the La autoantigen (Sjögren's syndrome antigen B) in B cells or the frontal brain causes extensive tissue loss. Mol Cell Biol 34: 123-131.

58. Triantafyllopoulou A, Tapinos N, Moutsopoulos HM (2004) Evidence for coxsackievirus infection in primary Sjögren's syndrome. Arthritis Rheum 50: $2897-2902$

59. Burfoot RK, Jensen CJ, Field J (2008) SNP mapping and candidate gene sequencing in the class I region of the HLA complex: searching for multiple sclerosis susceptibility genes in Tasmanians. Tiss Antigens 71: 42-50.

60. Vigneau S, Rohrlich PS, Brahic M, Bureau JF (2003) Tmevpg1, a candidate gene for the control of Theiler's virus persistence, could be implicated in the regulation of gamma interferon. J Virol 77: 5632-5638.

61. Sánchez Y, Huarte M (2013) Long non-coding RNAs: challenges for diagnosis and therapies. Nucleic Acid Ther 23: 15-20. 